\title{
Counting Schur rings over cyclic groups
}

\author{
Andrew Misseldine ${ }^{1}$
}

Received: 8 September 2015 / Accepted: 3 January 2019 / Published online: 22 January 2019

(c) Springer Science+Business Media, LLC, part of Springer Nature 2019

\begin{abstract}
Any Schur ring is uniquely determined by a partition of the elements of the group. An open question in the study of Schur rings is determining which partitions of the group induce a Schur ring. Although a structure theorem is available for Schur rings over cyclic groups, it is still a difficult problem to count all the partitions. For example, Kovacs, Liskovets, and Pöschel determine formulas to count the number of wreathindecomposable Schur rings. In this paper, we solve the problem of counting the number of all Schur rings over cyclic groups of prime power order and draw some parallels with Higman's PORC conjecture.
\end{abstract}

Keywords Schur ring · Cyclic group · Cyclotomic field · Catalan number · Schröder number $\cdot$ PORC conjecture

Mathematics Subject Classification 20c05 -05c25 -05e30 $\cdot 11 \mathrm{r} 18 \cdot 05 \mathrm{a} 15 \cdot 05 \mathrm{e} 15$

\section{Introduction}

Let $F$ be a field of characteristic zero, let $G$ denote a finite group, and let $F[G]$ denote the group algebra of $G$ with coefficients from $F$. For any subset $C \subseteq G$, let $\bar{C}:=\sum_{g \in C} g \in F[G]$. Such an element is called a simple quantity. Define $\alpha^{*}=\left(\sum_{g \in G} \alpha_{g} g\right)^{*}:=\sum_{g \in G} \alpha_{g} g^{-1}$ and $C^{*}:=\left\{x^{-1} \mid x \in C\right\}$ for all $\alpha \in F[G]$ and $C \subseteq G$. Let $\left\{C_{1}, C_{2}, \ldots, C_{r}\right\}$ be a partition of $G$, and let $S$ be the subspace of $F[G]$ spanned by the simple quantities $\overline{C_{1}}, \overline{C_{2}}, \ldots \overline{C_{r}}$. We say that $S$ is a Schur ring [22, Wielandt] over $G$ if

1. $C_{1}=\{1\}$,

2. For each $i$, there is a $j$ such that $C_{i}^{*}=C_{j}$,

3. For each $i$ and $j, \overline{C_{i}} \cdot \overline{C_{j}}=\sum_{k=1}^{r} \lambda_{i j k} \overline{C_{k}}$, for $\lambda_{i j k} \in F$.

Andrew Misseldine

andrewmisseldine@suu.edu

1 Southern Utah University, Cedar City, USA 
Schur rings were originally developed by Schur and Wielandt in the first half of the twentieth century. Schur rings were first used to study permutation groups, but in later decades applications of Schur rings have emerged in combinatorics, graph theory, and design theory $[8,14]$.

As mentioned above, any Schur ring is uniquely determined by a partition of the elements of the group, although not every partition determines a Schur ring. An open question in the study of Schur rings is determining which partitions of the group induce a Schur ring and which ones do not. Much work has been done to answer this question. In the case that our group $G$ is cyclic, a complete classification has been found [11,12]; see Theorem 2.1. In particular, the study of Schur rings over cyclic groups is a very active field with several recent papers being published on this topic: [9-12,17-19].

In this paper, we consider the problem of counting the number of Schur rings over $Z_{p^{n}}=\langle z\rangle$, the cyclic group of order $p^{n}$ where $p$ is a prime. Although a structure theorem is available for Schur rings over all cyclic groups, this still proves to be a difficult problem. Specializations of this problem have been considered before. For example, in [9] Kovács determines a formula to count the number of Schur rings over $Z_{2^{n}}$ which are wreath-indecomposable, that is, those Schur rings which cannot be properly factored as a wreath product of Schur rings (see page 6). Let $I(n, p)$ denote the number of wreath-indecomposable Schur rings over $Z_{p^{n}}$. Then Kovács' formula states: if $n \geq 3$ then:

$$
I(n, 2)=1+\frac{1}{3 \sqrt{17}} \sum_{k=0}^{n-1} a_{n-1-l}\left[\left(\frac{11+3 \sqrt{17}}{4}\right)^{k+1}-\left(\frac{11-3 \sqrt{17}}{4}\right)^{k+1}\right]
$$

where $a_{0}=2, a_{1}=-9$, and:

$$
a_{i}=\left(2 c_{i}-4 c_{i-1}\right)+\left(s_{i}-2 s_{i-1}\right)-3 \sum_{j=0}^{i-1} c_{i} s_{i-1-j}, \quad i \geq 2,
$$

where $c_{i}=\frac{1}{i+1}\left(\begin{array}{c}2 i \\ i\end{array}\right)$ and $s_{i}=\sum_{j=0}^{i} \frac{1}{j+1}\left(\begin{array}{c}2 j \\ j\end{array}\right)\left(\begin{array}{c}i+j \\ 2 j\end{array}\right)$ denote the $i$ th Catalan and Schröder number, respectively. In [13], Liskovets and Pöschel determine a simpler formula for wreath-indecomposable Schur rings over $Z_{p^{n}}$, where $p$ is an odd prime:

$$
I(n, p)=x c_{n-1},
$$

where $x$ denotes the number of divisors of $p-1$.

We present now the main results of this paper.

Theorem 1.1 The number of Schur rings over $Z_{p^{n}}$, where $p$ is an odd prime and $n \geq 2$, is given by the following recursive equation:

$$
\Omega(n)=x \Omega(n-1)+\sum_{k=2}^{n}\left(c_{k-1} x+1\right) \Omega(n-k),
$$


Table 1 Number of Schur rings over $Z_{p^{k}}$

\begin{tabular}{llllllll}
\hline$k \backslash p$ & 3 & 5 & 7 & 11 & 13 & 17 & 19 \\
\hline 1 & 2 & 3 & 4 & 4 & 6 & 5 & 6 \\
2 & 7 & 13 & 21 & 21 & 43 & 31 & 43 \\
3 & 25 & 58 & 113 & 113 & 313 & 196 & 313 \\
4 & 92 & 263 & 614 & 614 & 2288 & 1247 & 2288 \\
5 & 345 & 1203 & 3351 & 3351 & 16,749 & 7953 & 16,749 \\
6 & 1311 & 5531 & 18,329 & 18,329 & 122,675 & 50,775 & 122,675 \\
7 & 5030 & 25,511 & 100,372 & 100,372 & 898,706 & 324,323 & 898,706 \\
8 & 19,439 & 117,910 & 550,009 & 550,009 & $6,584,443$ & $2,072,078$ & $6,584,443$ \\
9 & 75,545 & 545,730 & $3,015,021$ & $3,015,021$ & $48,243,393$ & $13,239,896$ & $48,243,393$ \\
10 & 294,888 & $2,528,263$ & $16,531,326$ & $16,531,326$ & $353,479,684$ & $84,603,579$ & $353,479,684$ \\
\hline
\end{tabular}

Table 2 Number of Schur rings over $Z_{2^{n}}$

\begin{tabular}{lllllllllll}
\hline$n$ & 1 & 2 & 3 & 4 & 5 & 6 & 7 & 8 & 9 & 10 \\
\hline$\Omega(n)$ & 1 & 3 & 10 & 37 & 151 & 657 & 2989 & 14,044 & 67,626 & 332,061 \\
\hline
\end{tabular}

where $\Omega(0)=1, \Omega(1)=x$ denotes the number of divisors of $p-1$, and $c_{k}=$ $\frac{1}{k+1}\left(\begin{array}{c}2 k \\ k\end{array}\right)$ is the kth Catalan number.

The proof of Theorem 1.1 will be presented in Sect. 4. Table 1 lists the number of Schur rings over $Z_{p^{n}}$ up to the tenth power for the first seven odd primes.

Theorem 1.2 The number of Schur rings over $Z_{2^{n}}$, where $n \geq 4$, is given by the following recursive equation:

$$
\begin{aligned}
\Omega(n)= & \sum_{k=1}^{3} 2^{k} \Omega(n-k)-\left(c_{n-1}+s_{n-1}\right) \\
& +\sum_{k=4}^{n}\left(c_{k-1}+s_{k-1}-\sum_{j=1}^{k-3}\left(c_{j}+s_{j}\right)\right) \Omega(n-k)
\end{aligned}
$$

where $\Omega(0)=1, \Omega(1)=1, \Omega(2)=3, \Omega(3)=10, c_{k}=\frac{1}{k+1}\left(\begin{array}{c}2 k \\ k\end{array}\right)$ is the $k$ th Catalan number, and $s_{k}=\sum_{j=0}^{k} \frac{1}{j+1}\left(\begin{array}{c}2 j \\ j\end{array}\right)\left(\begin{array}{c}k+j \\ 2 j\end{array}\right)$ is the kth Schröder number.

The proof of Theorem 1.2 will be presented in Sect. 5. Table 2 lists the number of Schur rings over $Z_{2^{n}}$ up to the tenth power.

In [24] Ziv-Av enumerates all Schur rings over small finite groups up to order 63. There is a small intersection between the Schur rings over cyclic groups counted 
here and the Schur rings over those groups of small order enumerated by Ziv-Av, in particular cyclic groups of orders: 1, 2, 3, 4, 5, 7, 8, 9, 11, 13, 16, 17, 19, 23, 25, 27, $29,31,32,27,41,43,47,49,53,59$, and 61 . In all instances, the two enumerations agree.

Higman's PORC conjecture states that for any fixed $n$, the number of isomorphism classes of groups of order $p^{n}$ is a PORC (polynomial on residue classes) function of $p$, that is, the counting function acts like a polynomial dependent on the prime $p$. The conjecture has been verified for $n \leq 7$ [20,21]. Although Higman's PORC conjecture is likely untrue for $n \geq 10$ [2], various versions of the PORC conjecture have been proven for specific families of $p$-groups, e.g., $[3,5,6,23]$. In the language of the PORC conjecture, Theorem 1.1 shows that the number of Schur rings over a cyclic $p$-group is a PORC function for all $n$, as the polynomials $\Omega(n)$ depend only on powers of $p$ and the number of divisors of $p-1$. It is conceivable to extend the PORC conjecture to the counting of Schur rings over $p$-groups, for which it would be interesting to know which partial truths hold besides cyclic $p$-groups.

\section{Schur rings}

Let $S$ be a Schur ring over the finite group $G$ associated with the partition $\left\{C_{1}, C_{2}, \ldots, C_{r}\right\}$. The subsets $C_{1}, \ldots, C_{r}$ are called the primitive sets of $S$ or $S$ classes. Let $\mathcal{D}(S)$ denote the set of $S$-classes. If $C \subseteq G$ and $\bar{C} \in S$, then $C$ is called an $S$-set. If $C$ is also a subgroup of $G$, then we say that $C$ is an $S$-subgroup of $G$. If $H$ is an $S$-subgroup, then let $S_{H}:=\operatorname{Span}_{F}\left\{\overline{C_{i}} \mid C_{i} \subseteq H\right\}$. It is clear that $S_{H}$ is a Schur ring over $H$.

Define the Hadamard product (or circle product) on $F[G]$ as $\left(\sum_{g \in G} \alpha_{g} g\right) \circ$ $\left(\sum_{g \in G} \beta_{g} g\right):=\sum_{g \in G} \alpha_{g} \beta_{g} g$. It is well known that a subring of $F[G]$ is a Schur ring if and only if it is closed under $*$ and $\circ$ and contains the elements 1 and $\bar{G}$ (see [19] Lemma 1.3 for a proof). It is immediate that if $S$ and $T$ are Schur rings over $G$, then $S \cap T$ is likewise a Schur ring over $G$.

Every finite group algebra $F[G]$ is a Schur ring, resulting from the partition consisting of only singletons. The partition $\{\{1\}, G \backslash\{1\}\}$ induces the trivial Schur ring over $G$, denoted $F[G]^{0}$.

Suppose $\mathcal{H} \leq \operatorname{Aut}(G)$ and define $F[G]^{\mathcal{H}}:=\{\alpha \in F[G] \mid \sigma(\alpha)=\alpha$, for all $\sigma \in$ $\mathcal{H}$, the maximal subalgebra of $F[G]$ fixed by $\mathcal{H}$. Then $F[G]^{\mathcal{H}}$ is a Schur ring corresponding to the partition of orbits of the $\mathcal{H}$-action ${ }^{1}$ on $G$. These Schur rings are called orbit $S$ chur rings, and the sum of an $\mathcal{H}$-orbit is called an $\mathcal{H}$-period. The center of $F[G]$

\footnotetext{
${ }^{1}$ The notation $F[G]^{\mathcal{H}}$ for orbit Schur rings should not be confused with the notation for the trivial Schur ring $F[G]^{0}$, despite their similar superscripts. Zero was used to mark the trivial Schur ring simply because trivial mathematical statements are often denoted by the trivial number, 0 , and since there is no natural interpretation of the phrase "zero automorphism" in the group theory (other than the trivial group itself) to confuse 0 as an automorphism subgroup. It is possible for the trivial Schur ring to coincide with an orbit ring, such as in the case of the cyclic group of prime order; this should lead to no confusion. See the proof of Corollary 2.3 .
} 
is an orbit Schur ring with $\mathcal{H}=\operatorname{Inn}(G)$. Let $F[G]^{\operatorname{Aut}(G)}$ be called the rational ${ }^{2}$ Schur ring, whose primitive sets are the automorphism classes of $G$. In the case that $G$ is cyclic, these automorphism classes are exactly the sets of elements which have the same order. For an abelian group $G$, let $F[G]^{\langle *\rangle}$ be called the symmetric Schur ring, whose primitive sets are the inverse classes of $G$, denoted $F[G]^{ \pm}$.

Let $S$ and $T$ be Schur rings over $F[G]$ and $F[H]$, respectively. We naturally can view $G$ and $H$ as subgroups of $G \times H$. Define $S \cdot T:=\operatorname{Span}_{F}\{\bar{C} \cdot \bar{D} \mid C \in \mathcal{D}(S), D \in$ $\mathcal{D}(T)\}$, called the dot product of $S$ and $T$. This forms a Schur ring with partition $\mathcal{D}(S \cdot T)=\{C D \subseteq G \times H \mid C \in \mathcal{D}(S), D \in \mathcal{D}(T)\}$. Furthermore, $S \cdot T \cong S \otimes_{F} T$, as $F$-algebras. Because of this fact, the Schur ring $S \cdot T$ is often called the tensor product of Schur rings.

The notion of wedge product of Schur rings presented below is originally due to Leung and Man [12]. Suppose $H \unlhd G$, and let $S$ be a Schur ring over $H$ and $T$ a Schur ring over $G / H$. Let $\pi: G \rightarrow G / H$ be the natural quotient map. Then define $S$ ₹ $T:=S+\pi^{-1}(T)$, called the wreath product of $S$ and $T$. The wreath product is likewise a Schur ring with partition $\mathcal{D}\left(S \_T\right)=\mathcal{D}(S) \cup\left\{\pi^{-1}(C) \mid C \in \mathcal{D}(T), C \neq\right.$ $\{1\}\}$. It follows that $(S<T)_{H}=S$ and $\pi(S<T)=T$.

Let $1<K \leq H<G$ be a sequence of finite groups such that $K \unlhd G$. Let $S$ be a Schur ring over $H$ and $T$ a Schur ring over $G / K$. Let $\pi: G \rightarrow G / K$ be the quotient map. Then define $S \wedge_{K} T:=S+\pi^{-1}(T)$, which denotes the wedge product of $S$ and $T$. (When the context is clear, the subscript may be omitted.) If we assume that $H / K$ is a $T$-subgroup, $K$ is an $S$-subgroup, and $\pi(S)=T_{H / K}$, then $S \wedge T$ is a Schur ring over $G$ with partition $D(S \wedge T)=D(S) \cup\left\{\pi^{-1}(C) \mid C \in \mathcal{D}(T) \backslash \mathcal{D}\left(T_{H / K}\right)\right\}$. Like above, it follows that $(S \wedge T)_{H}=S$ and $\pi(S \wedge T)=T$. If $H=K$, then $S \wedge T=S_{\imath} T$. Thus, the wedge product of Schur rings is a generalized wreath product of Schur rings, although the special case of wreath products will need particular attention below.

Let $S$ be a Schur ring over $G$. If there exists subgroups $1<K \leq H<$ $G$, with $K \unlhd G$, and Schur rings $R$ and $T$ over $H$ and $G / K$, respectively, such that $S=R \wedge_{K} T$, then we say that $S$ is wedge-decomposable; otherwise, we say that $S$ is wedge-indecomposable. If $S$ is wedge-decomposable, we call $1<K \leq H<G$ a wedge-decomposition of $S$. We define the terms wreathdecomposable, wreath-indecomposable, and wreath-decomposition analogously. Note every wreath-decomposable Schur ring is clearly wedge-decomposable and not every wreath-indecomposable Schur ring is wedge-decomposable.

Leung and Man used the constructions of Schur rings mentioned above to classify all Schur rings over $Z_{m}$.

Theorem 2.1 [11,12] Suppose $G=Z_{m}$ and $S$ is a Schur ring over $G$. Then $S$ is trivial, an orbit ring, a dot product of Schur rings, or a wedge product of Schur rings.

The following corollaries are immediate from Theorem 2.1.

Corollary 2.2 Suppose $G=Z_{p^{n}}$, for some prime $p$, and $S$ is a Schur ring over $G$. Then $S$ is trivial, an orbit ring, or a wedge product of Schur rings.

\footnotetext{
2 Rational Schur rings are so called because they are the Schur rings which are fixed by all automorphisms in the same way the rational field is fixed by all automorphisms in Galois theory.
} 
Corollary 2.3 Let $G=Z_{p}$, for some prime $p$, and let $S$ be a Schur ring over $G$. Then $S$ is an orbit Schur ring.

Corollary 2.4 Suppose $G=Z_{p^{n}}$. Then for any wedge-decomposable Schur ring $S$ over $G$, there exists a wedge-decomposition $1<K \leq H<G$ such that $S_{H}$ is a wedge-indecomposable orbit algebra or a trivial Schur ring over $H$.

\section{A correspondence between Schur rings and cyclotomic fields}

Let $p$ be a prime number, let $G_{n}:=\mathbb{Z}_{p^{n}}$, and let $\mathcal{K}_{n}:=\mathbb{Q}\left(\zeta_{n}\right)$, where $\zeta_{n}$ is a complex primitive $p^{n}$ th root of unity. For $0 \leq k \leq n$, let $L_{k}$ denote the set of subfields of $\mathcal{K}_{k}$ which are not subfields of $\mathcal{K}_{k-1}$, called the kth layer of the lattice of subfields of $\mathcal{K}_{n}$. Let $\omega: \mathbb{Q}\left[G_{n}\right] \rightarrow \mathcal{K}_{n}$ be the algebra epimorphism given by $z \mapsto \zeta$.

The epimorphism $\omega$ likewise maps each Schur ring over $G_{n}$ onto a subfield of $\mathcal{K}_{n}$. The map $\omega$ restricts to a lattice-isomorphism ${ }^{3}$ between the orbit Schur rings over $G_{n}$ and the subfields of $\mathcal{K}_{n}$, that is,

$$
\omega\left(\mathbb{Q}\left[G_{n}\right]^{\mathcal{H}}\right)=\mathcal{K}_{n}^{\mathcal{H}} .
$$

A consequence of this is that $\mathbb{Q}\left[G_{n}\right]^{\mathcal{H}_{1}}=\mathbb{Q}\left[G_{n}\right]^{\mathcal{H}_{2}}$ if and only if $\mathcal{H}_{1}=\mathcal{H}_{2}$, a statement that may fail for non-cyclic groups. For example, suppose $G=Z_{4} \times Z_{2}=$ $\langle a, b\rangle$ and

$$
S=\operatorname{Span}_{\mathbb{Q}}\left\{1, a^{2}, b+a^{2} b, a+a^{3}+a b+a b^{3}\right\} \cong \mathbb{Q} Z_{2} 2 \mathbb{Q} Z_{2} 2 \mathbb{Q} Z_{2} .
$$

In fact, $S=\mathbb{Q}[G]^{\operatorname{Aut}(G)}$. The automorphism group $\operatorname{Aut}(G)$ is given by:

$$
\operatorname{Aut}(G)=\left\langle\sigma: \underset{b \mapsto a^{2} b}{a \mapsto a}, \tau: \begin{array}{c}
a \mapsto a^{3} b \\
b \mapsto a^{2} b
\end{array}\right\rangle \cong D_{4} \text {. }
$$

Let $\mathcal{H}=\langle\tau\rangle \lesseqgtr \operatorname{Aut}(G)$. It can be shown that $\mathbb{Q}[G]^{\mathcal{H}}=\mathbb{Q}[G]^{\operatorname{Aut}(G)}$, although $\mathcal{H} \neq \operatorname{Aut}(G)$.

It is also clear to see that:

$$
\omega\left(\mathbb{Q}\left[G_{n}\right]^{0}\right)=\mathbb{Q},
$$

since $\mathbb{Q}\left[G_{n}\right]^{0} \leq \mathbb{Q}\left[G_{n}\right]^{\operatorname{Aut}\left(G_{n}\right)} \mapsto \mathbb{Q}$.

It remains to examine where the wreath-decomposable Schur rings ${ }^{4}$ land under $\omega$. Suppose $G=G_{n}$, and $S=S_{H} \wedge S_{G / K}$ is a wedge-decomposable Schur ring over $\mathbb{Q}[G]$ with wedge-decomposition $1<K \leq H<G$. For any $C \in \mathcal{D}(S) \backslash \mathcal{D}\left(S_{H}\right)$, we see that $\bar{C}$ is a union of cosets of $K$ and so $\omega(\bar{C})=0$, as ker $\omega$ is the principal ideal

\footnotetext{
3 This isomorphism still holds, as well as Eqs. (3.1)-(3.3), even when the cyclic group has arbitrary order.

4 Although the general case would also involve dot products, dot product Schur rings do not arise over $Z_{p^{n}}$ since there is no direct product structure here. In any case, $\omega(S \cdot T)=\omega(S) \vee \omega(T)$, the compositum field of $\omega(S)$ and $\omega(T)$ inside of $\mathbb{Q}\left(\zeta_{m}\right)$. Of course, $\omega(S) \vee \omega(T) \cong \omega(S) \otimes \mathbb{Q} \omega(T)$.
} 
$\left(\overline{G_{1}}\right)$ and $G_{1} \leq K$. Therefore, $\omega(S)=\omega\left(S_{H}+\pi^{-1}\left(S_{G / K}\right)\right) \subseteq \omega\left(S_{H}\right)$, which proves that

$$
\omega\left(S_{H} \wedge S_{G / K}\right)=\omega\left(S_{H}\right)
$$

Lemma 3.1 For any prime $p, \mathbb{Q}\left[Z_{p^{n}}\right]^{\mathcal{G}_{n}}=2_{k=1}^{n} \mathbb{Q}\left[Z_{p}\right]^{0}$.

Proof The automorphism classes in $G_{n}$ have the form $G_{k} \backslash G_{k-1}$, where $0 \leq k \leq n$, and $G_{k} \backslash G_{k-1}$ is the union of all cosets of $G_{k-1}$ except $G_{k-1}$ itself.

Theorem 3.2 Let $S$ be a Schur ring over $G_{n}$ such that $\omega(S)=\mathbb{Q}$. Then there exists a subgroup $H \leq G_{n}$ and a Schur ring $T$ over $G_{n} / H$ such that $S=\mathbb{Q}[H]^{0} \imath T$.

Proof Let $G=G_{n}$. By Corollary 2.2, $S$ is trivial, an orbit ring, or wedgedecomposable. If $S$ is trivial, then we are done. In the second case, $\mathbb{Q}[G]^{\operatorname{Aut}(G)}$ is the unique Schur ring which maps onto $\mathbb{Q}$, which has the desired form by Lemma 3.1. Finally, suppose there exist $S$-subgroups $1<K \leq H<G$ such that $S=S_{H} \wedge S_{G / K}$ and $S_{H}$ is trivial or a wedge-indecomposable orbit Schur ring. But $\omega\left(S_{H}\right)=\omega(S)=$ $\mathbb{Q}$. Since the only indecomposable orbit ring which maps onto $\mathbb{Q}$ is $S_{H}=\mathbb{Q}\left[G_{1}\right]^{0}$, again by Lemma 3.1, $S_{H}=\mathbb{Q}[H]^{0}$ in either case. Since $K$ is a non-trivial $S_{H^{-}}$ subgroup, it must be that $K=H$. Therefore, $S=S_{H} 2 S_{G / H}=\mathbb{Q}[H]^{0} 2 S_{G / H}$.

Theorem 3.3 Let $S$ be a Schur ring over $G_{n}$ and $\omega(S) \in L_{k}$ for $0<k<n$. Then $S$ is wedge-decomposable.

Proof If $S$ is not a wedge product, then it is trivial or an orbit Schur ring. As the trivial Schur ring maps into $L_{0}=\{\mathbb{Q}\}, S$ is not trivial. Suppose $S=\mathbb{Q}\left[G_{n}\right]^{\mathcal{H}}$ for some $\mathcal{H} \leq \operatorname{Aut}\left(G_{n}\right)$. Let $g \in G_{n}$ be an element of order $p^{m}$ where $k<m \leq n$. As the set $\left\{1, \zeta_{m}, \zeta_{m}^{2}, \ldots, \zeta_{m}^{p-1}\right\}$ is linearly independent over $\mathcal{K}_{m-1}$, then it is immediate that:

$$
\omega\left(\sum_{\sigma \in \mathcal{H}} \sigma(g)\right) \in \mathcal{K}_{m-1} \text { if and only if } \omega\left(\sum_{\sigma \in \mathcal{H}} \sigma(g)\right)=0 .
$$

As $\operatorname{ker} \omega=\left(\overline{G_{1}}\right)$, we see that $\omega\left(\sum_{\sigma \in \mathcal{H}} \sigma(g)\right)=0$ if and only if $\sum_{\sigma \in \mathcal{H}} \sigma(g)$ is a sum of $G_{1}$-cosets. Thus, all the $S$-classes over $G_{n} \backslash G_{k}$ are union of cosets of $G_{1}$. Thus, $S$ is wedge-decomposable with decomposition $1<G_{1} \leq G_{k}<G_{n}$, a contradiction.

Theorem 3.4 Let $S$ be a Schur ring over $G_{n}$ such that $\omega(S) \in L_{n}$. Then $S$ is an orbit Schur ring, and hence, $S$ is the unique Schur ring over $G_{n}$ which maps to $\omega(S)$.

Proof By (3.2) and (3.3) if $S$ is trivial or wedge-decomposable then $\omega(S)$ is not in the top layer. Thus, $S$ is an orbit Schur ring. As $\omega$ is injective on the orbit Schur rings, $S$ is the unique orbit Schur ring mapping onto $\omega(S)$.

Corollary 3.5 Let $S$ be a Schur ring over $G_{n}$. If $G_{m}$ is an $S$-subgroup, then

$$
\omega(S) \cap \mathcal{K}_{m}=\omega\left(S_{G_{m}}\right)
$$


Proof The result is immediate if $S$ is trivial. If $S$ is an orbit Schur ring, then $\omega(S) \cap$ $\mathcal{K}_{m}=\omega\left(S \cap G_{m}\right)=\omega\left(S_{H}\right)$ follows from the lattice-isomorphism. If $S$ is wedgedecomposable over $1<G_{1} \leq H<G_{n}$, then $S_{H}$ can be assumed to be trivial or an orbit Schur ring and $\omega(S) \cap \mathcal{K}_{m}=\omega\left(S_{H}\right) \cap \mathcal{K}_{m}=\omega\left(\left(S_{H}\right)_{G_{m}}\right)=\omega\left(S_{G_{m}}\right)$.

\section{Counting Schur rings over cyclic $p$-groups}

Let $\Omega(n)$ denote the number of Schur rings over $G_{n}$, and let $\Omega(n, k)$ denote the number of Schur rings $S$ over $G_{n}$ such that $\omega(S)=\mathcal{K}_{k}$. We have that $\Omega(0)=1$ since there is exactly one Schur ring over $G_{0}=1$, the group ring itself. Also, if $x$ denotes the number of divisors of $p-1$, then $\Omega(1)=x$ by Corollary 2.3.

Lemma 4.1 The number of Schur rings over $G_{n}$, for any prime $p$ and $n \geq 1$, mapping onto $\mathbb{Q}$ with respect to $\omega$ is equal to the sum of the number of $S$ chur rings over $G_{k}$ for $0 \leq k \leq n-1$, that is,

$$
\Omega(n, 0)=\sum_{k=0}^{n-1} \Omega(k) .
$$

Proof Let $G=G_{n}$. By Theorem 3.2, if $\omega(S)=\mathbb{Q}$ then $S=\mathbb{Q}\left[G_{k}\right]^{0} 2 T$ for some Schur ring $T$ over $G / G_{k}$. If we consider the trivial Schur ring on $G$ as a trivial wreath product, that is, $\mathbb{Q}[G]^{0}=\mathbb{Q}[G]^{0}>\mathbb{Q}[1]$, then every Schur ring descending to $\mathbb{Q}$ has the form $S=\mathbb{Q}\left[G_{k}\right]^{0} \imath T$, where $1 \leq k \leq n$ and $T$ ranges over all the Schur rings of $G / G_{k} \cong G_{n-k}$. Since every Schur ring over $G$ of this form maps to $\mathbb{Q}$, the proof is finished.

For the remainder of this section, we will assume that $p$ is an odd prime.

Lemma 4.2 For an odd prime $p$, the number of Schur rings over $G_{n}$ mapping to $\mathcal{K}_{1}$ with respect to $\omega$ is equal to the number of Schur rings over $G_{n-1}$, that is,

$$
\Omega(n, 1)=\Omega(n-1) .
$$

Proof Let $G=G_{n}$. If $n=1$, then $\Omega(n-1)=\Omega(0)=1$. By Corollary 2.3, there is only one Schur ring which maps to $\mathcal{K}_{1}$. So the result follows.

Suppose that $n \geq 2$. Let $S$ be the orbit Schur $\operatorname{ring}$ over $G$ which maps onto $\mathcal{K}_{1}$. By Theorem 3.3, $S$ is wedge-decomposable. By Corollary 2.4, there is a wedgedecomposition of $S, 1<K \leq H<G$, such that $S_{H}$ is trivial or an indecomposable orbit Schur ring. If $S_{H}$ is trivial, then $\omega(S)=\omega\left(S_{H}\right)=\mathbb{Q}$ by (3.2) and (3.3). Thus, $S_{H}$ is an indecomposable orbit Schur ring where $K=H=G_{1}$ by Theorem 3.3. In fact, $S_{H}=\mathbb{Q}\left[G_{1}\right]$. This shows that $S=\mathbb{Q}\left[G_{1}\right] \imath T$, where $T$ is some Schur ring over $G / G_{1}$. Since every Schur ring over $G$ of this form maps to $\mathcal{K}_{1}$, the proof is finished.

Lemma 4.3 For an odd prime $p$ and $n \geq 2$, the number of Schur rings over $G_{n}$ mapping to $\mathcal{K}_{k}$ for $1<k \leq n$ with respect to $\omega$ is equal to the sum of the number of 
Schur rings over $G_{n-1}$ mapping onto $\mathcal{K}_{j}$ where $j$ ranges between $k-1$ and $n-1$, that is,

$$
\Omega(n, k)=\sum_{j=k-1}^{n-1} \Omega(n-1, j) .
$$

Note that when $k=n,(4.3)$ simplifies to:

$$
\Omega(n, n)=1
$$

Proof Let $G=G_{n}$. Let $S$ be a Schur ring such that $\omega(S)=\mathcal{K}_{k}$. Since $\mathcal{K}_{j}$ is a field in the top layer of $\mathcal{L}_{j}$; the identity $\Omega(j, j)=1$ follows immediately from Theorem 3.4 for all $j$. In particular, $\Omega(n, n)=1=\Omega(n-1, n-1)$, that is, (4.3) holds for $k=n$.

If $1<k<n$, then $S$ is wedge-decomposable by Theorem 3.3. In particular, there exists a wedge-decomposition such that $1<K \leq H=G_{k}<G$ and $S=\mathbb{Q}[H] \wedge T$, where $T$ is a Schur ring over $G / K$. As $\overline{G_{1}} \in \mathbb{Q}[H]$ and $G_{1}$ is the unique minimal subgroup of $G$, we may assume $K=G_{1}$, that is, all $S$-classes outside of $H$ are unions of cosets of $G_{1}$. Let $\pi: G \rightarrow G / K \cong G_{n-1}$ be the natural map. Then $\pi\left(\left.S\right|_{H}\right)=$ $\mathbb{Q}[H / K] \cong \mathbb{Q}\left[G_{k-1}\right]$. Therefore, the wedge product $\mathbb{Q}[H] \wedge T$ is possible if and only if $T_{H / K}=\mathbb{Q}[H / K]$. By Corollary 3.5, we have $\mathcal{K}_{k-1}=\omega\left(T_{H / K}\right)=\omega(T) \cap \mathcal{K}_{k-1}$. This is only possible if $\omega(T)=\mathcal{K}_{j}$ for some $k-1 \leq j \leq n-1$. Since every Schur ring of this type can be wedged to $\mathbb{Q}[H]$, the equality is proven.

By mimicking the proofs of Lemmas 4.2 and 4.3 but using arbitrary subfields of $\mathcal{K}_{n}$, we can prove the following proposition.

Lemma 4.4 Let $E, F \in L_{k}$. For an odd prime $p$, the number of Schur rings over $G_{n}$ which map onto $E$ with respect to $\omega$ is equal to the number of Schur rings over $G_{n}$ which map onto $F$. In particular, the number of Schur rings mapping onto $E$ is equal to $\Omega(n, k)$.

Proof of Theorem 1.1 Basic Galois theory shows that the lattice of fields in $L_{k}$ for $2 \leq k \leq n$ and the lattice $L_{1} \cup L_{0}$ are both lattice-isomorphic to the divisor lattice of $p-1$. Thus, these lattices contain $x$ many fields. Of course, $\left|L_{0}\right|=1$ and $\left|L_{1}\right|=x-1$. The equation:

$$
\Omega(n)=\Omega(n, 0)+(x-1) \Omega(n, 1)+x \sum_{k=2}^{n} \Omega(n, k)
$$

then follows from Lemma 4.4.

Using (4.1) and (4.2), we can rewrite (4.5) as:

$$
\Omega(n)=x \Omega(n-1)+\sum_{k=0}^{n-2} \Omega(k)+x \sum_{k=2}^{n} \Omega(n, k) .
$$


Thus, we need to expand $\sum_{k=2}^{n} \Omega(n, k)$ using (4.3). This will produce an equation of the following form:

$$
\sum_{k=2}^{n} \Omega(n, k)=\sum_{k=1}^{n-1} c_{k} \Omega(n-k, 1)=\sum_{k=2}^{n} c_{k-1} \Omega(n-k)
$$

for some positive integers $c_{k}$. In particular, the $j$ th iteration of (4.3) will produce an equation of the form:

$$
\sum_{k=2}^{n} \Omega(n, k)=\sum_{i=1}^{j-1} c_{i} \Omega(n-i, 1)+\sum_{k=j+1}^{n} c_{j k} \Omega(n-j, k-j)
$$

for some positive integers $c_{j k}$. We note that $c_{i(i+1)}=c_{i}$ and $c_{0 k}=1$ for all $k$. Furthermore,

$$
c_{j k}=\sum_{\ell=j}^{k} c_{(j-1) \ell}
$$

by Lemma 4.3 . When $0<j<k-1$, the above equation can be rewritten recursively to give:

$$
c_{j k}=c_{(j-1) k}+\sum_{\ell=j}^{k-1} c_{(j-1) \ell}=c_{(j-1) k}+c_{j(k-1)} .
$$

From above, we can create a triangular array of integers where $k$ indexes the rows $(k \geq 1)$ and $j$ indexes the columns $(0 \leq j<k)$. The diagonal entries of the triangle give the values of $c_{i}$. For convenience, we define $c_{00}=1$ and $c_{j j}=c_{(j-1) j}$ for $j>0$. This extended triangular array is known as Catalan's triangle. One property of Catalan's triangle is that the sequence of diagonal entries is the sequence of Catalan numbers [15].

Equation (1.3) then follows immediately from (4.6) and (4.7), and the previous observation about the Catalan numbers.

Let $\mathcal{F}(z)=\sum_{n=0}^{\infty} \Omega(n) z^{n}$ be the generating function of $\Omega$. Using the usual generating function calculations, including the generating function for the Catalan numbers, one computes that:

$$
\mathcal{F}(z)=\frac{2(1-z)}{-2 z^{2}+(x-2) z-(x-2)+x(1-z) \sqrt{1-4 z}}
$$

Now, one can continue working with the generating function of $\Omega(n)$ using the typical combinatorial methods to produce a non-recursive formula. Unfortunately, this formula is highly complicated and much less practical than the recursive version (1.3). 
Treating $x$ as a variable, $\Omega(n)$ is a monic degree $n$ polynomial whose coefficients asymptotically grows at the same rate as $\left(\begin{array}{l}n \\ k\end{array}\right)$. An asymptotic estimate for $\Omega(n)$ is then given as:

$$
\Omega(n) \sim \sum_{k=0}^{n}\left(\begin{array}{l}
n \\
k
\end{array}\right) x^{n-k}=(x+1)^{n} .
$$

The rate of convergence of the sequence $\left\{\frac{\Omega(n)}{(x+1)^{n}}\right\}_{n}$ toward its limit increases as $x$ increases.

\section{Counting Schur rings over cyclic 2-groups}

We consider now when $p=2$. Note that $\Omega(0)=1, \Omega(1)=1$, and (4.1) still holds for $p=2$. Additionally, Lemma 4.3 remains true for $p=2$ when $n=k=2$ or for all $n \geq 3$ and $2<k \leq n$ by the same proof. (The change of bounds is due to the considerations of possible wedge products.) From these, we can compute $\Omega(2)=3$. Lemma 4.2 no longer applies since there is no first layer, that is, $L_{1}=\emptyset$. Instead, we will treat $k=2$ as the base case in the recurrence relation on $\Omega(n, k)$.

Lemma 5.1 For $p=2$ and $n \geq 3$, the number of Schur rings over $G_{n}$ mapping to $\mathcal{K}_{2}$ with respect to $\omega$ is equal to the difference between number of Schur rings over $G_{n-1}$ and the number of Schur rings over $G_{n-2}$ mapping onto $\mathbb{Q}$, that is,

$$
\Omega(n, 2)=\Omega(n-1)-\Omega(n-2,0) .
$$

Proof Let $S$ be a Schur ring over $G_{n}$ such that $\omega(S)=\mathcal{K}_{2}=\mathbb{Q}(i)$. Since $n \geq 3$, it must be that $S$ is wedge-decomposable of the form $S=\mathbb{Q}\left[G_{2}\right] \wedge T$ for some Schur ring $T$ over $G_{n-1}$ such that $T_{G_{1}}=\mathbb{Q}\left[G_{1}\right]$, by the same reasoning used in Lemma 4.3. Now, every Schur ring over $G_{n-1}$ has this property except those of the form $T=\mathbb{Q}\left[G_{k}\right]^{0} 2 T^{\prime}$ for $1<k \leq n-1$. Now, there are exactly $\Omega(n-2,0)$ such Schur rings by Lemma 4.1. Therefore, the result follows.

From (5.1) and the other established identities, we can compute that $\Omega(3)=10$.

Unlike the odd case, basic Galois theory shows that, for $p=2,\left|L_{2}\right|=1$, and $\left|L_{k}\right|=3$ for all $3 \leq k \leq n$. Note that $L_{n}$ contains three fields: $\mathcal{K}_{k}=\mathbb{Q}\left(\zeta_{2^{k}}\right)$, $\mathcal{S}_{k}:=\mathbb{Q}\left(\zeta_{2^{k}}+\zeta_{2^{k}}^{-1}\right)$, and $\mathcal{S}_{k}^{\prime}:=\mathbb{Q}\left(\zeta_{2^{k}}-\zeta_{2^{k}}^{-1}\right)$. This major difference comes from the fact that when $p$ is odd $\mathcal{K}_{n}$ has a cyclic Galois group and the Galois group is noncyclic when $p=2$. A consequence of this fact is that Lemma 4.4 fails for $p=2$. For example, the number of Schur rings over $G_{4}=Z_{16}$ which map onto $\mathcal{K}_{3}=\mathbb{Q}\left(\zeta_{8}\right)$ is three, but the number of Schur rings mapping onto $\mathbb{Q}\left(\zeta_{8}+\zeta_{8}^{-1}\right)$ is four. Let $\Omega_{\mathcal{S}}(n, k)$ be the number of Schur rings over $G_{n}=Z_{2^{n}}$ which map onto $\mathbb{Q}\left(\zeta_{2^{n}}+\zeta_{2^{n}}^{-1}\right)$ via $\omega$.

Lemma 5.2 For $p=2$, the number of Schur rings over $G_{n}$ mapping onto $\mathcal{S}_{k}$ via $\omega$ is the same as the number of Schur rings mapping onto $\mathcal{S}_{k}^{\prime}$. 
Proof If $\mathcal{S}_{k}$ and $\mathcal{S}_{k}^{\prime}$ are in the top layer, then there is exactly one Schur ring mapping onto each field by Theorem 3.4, namely $\mathbb{Q}\left[G_{k}\right]^{\left\langle 2^{k}-1\right\rangle}$ and $\mathbb{Q}\left[G_{k}\right]^{\left\langle 2^{k-1}-1\right\rangle}$, respectively. Otherwise, each Schur ring mapping onto these fields must be wedge-decomposable. Let $\pi: G_{n} \rightarrow G_{n-1}$ be the natural quotient map. Then $\pi\left(\mathbb{Q}\left[G_{k}\right]^{\left\langle 2^{k}-1\right\rangle}\right)=$ $\mathbb{Q}\left[G_{k-1}\right]^{\left\langle 2^{k-1}-1\right\rangle}=\pi\left(\mathbb{Q}\left[G_{k}\right]^{\left\langle 2^{k-1}-1\right\rangle}\right)$. Since the images are the same, the number of possible wedge products for the two cases is the same.

Lemma 5.3 For $p=2$ and all $n$,

$$
\Omega_{\mathcal{S}}(n, n)=1
$$

and, for $3<k<n$,

$$
\Omega_{\mathcal{S}}(n, k)=\Omega_{\mathcal{S}}(n-1, k-1)+2 \sum_{j=k}^{n-1} \Omega_{\mathcal{S}}(n-1, j) .
$$

Proof Equation (5.2) is immediate from Theorem 3.4. Assume $3<k<n$. Because of the possible wedge-decompositions, it suffices to count the number of Schur rings $T$ over $G_{n-1}$ for which $T_{G_{k-1}}=\mathbb{Q}\left[G_{k-1}\right]^{\left\langle 2^{k-1}-1\right\rangle}$. This is exactly the number of Schur rings over $G_{n-1}$ which map onto $\mathcal{S}_{j}$ for $k-1 \leq j \leq n-1$ or onto $\mathcal{S}_{j}^{\prime}$ for $k \leq j \leq n-1$. The result then follows from Lemma 5.2.

Lemma 5.4 For $p=2$ and $n>3$,

$$
\Omega_{\mathcal{S}}(n, 3)=\Omega(n-1,2)+2 \sum_{j=3}^{n-1} \Omega_{\mathcal{S}}(n-1, j) .
$$

Proof Again, because of wedge-decompositions, it suffices to count the number of Schur rings $T$ over $G_{n-1}$ for which $T_{G_{2}}=\mathbb{Q}\left[G_{1}\right]<\mathbb{Q}\left[G_{1}\right]$. This includes the Schur rings over $G_{n-1}$ which map onto $\mathcal{S}_{j}$ or onto $\mathcal{S}_{j}^{\prime}$ for $3 \leq j \leq n-1$. On the other hand, no Schur ring which maps onto $\mathcal{K}_{j}$ has this property for $j>1$. It remains to examine which Schur rings that map onto $\mathbb{Q}$ have this property. By Theorem 3.2, if $\omega(T)=\mathbb{Q}$, then $T=\mathbb{Q}\left[G_{1}\right] \prec T^{\prime}$ for some Schur ring $T^{\prime}$ over $G_{n-2}$ such that $T_{G_{1}}^{\prime}=\mathbb{Q}\left[G_{1}\right]$. As was seen in the proof of Lemma 5.1, the number of choices for $T^{\prime}$ is $\Omega(n-1,2)$. The result then follows from Lemma 5.2.

Proof of Theorem 1.2 The equation:

$$
\Omega(n)=\Omega(n, 0)+\Omega(n, 2)+\sum_{k=3}^{n}\left(\Omega(n, k)+2 \Omega_{\mathcal{S}}(n, k)\right) .
$$

is immediate from Lemma 5.2 and the number of fields in $L_{k}$, as discussed above. Considering the discussion of Catalan's triangle in the proof of Theorem 1.1, (5.5) can be rewritten as: 


$$
\Omega(n)=\Omega(n, 0)+\sum_{k=0}^{n-2} c_{k} \Omega(n-k, 2)+2 \sum_{k=3}^{n} \Omega_{\mathcal{S}}(n, k)
$$

Therefore, we seek to express $2 \sum_{k=3}^{n} \Omega_{\mathcal{S}}(n, k)$ in terms of the $\Omega(n, k)$. Using (5.3) and (5.4), we obtain:

$$
2 \sum_{k=3}^{n} \Omega_{\mathcal{S}}(n, k)=\sum_{i=1}^{n-2} s_{i} \Omega(n-i, 2)
$$

for some positive integers $s_{i}$. In particular, the $j$ th iteration of (5.3) and (5.4) will produce an equation of the form:

$$
2 \sum_{k=3}^{n} \Omega_{\mathcal{S}}(n, k)=\sum_{i=1}^{j-1} s_{i} \Omega(n-i, 2)+\sum_{k=j+1}^{n} s_{j k} \Omega_{\mathcal{S}}(n-j, k-j)
$$

for some positive integers $s_{j k}$. We note that $s_{i(i+1)}=s_{i}$ and $s_{0 k}=1$ for all $k$. Furthermore,

$$
s_{j k}=s_{(j-1) k}+2 \sum_{\ell=j}^{k-1} s_{(j-1) \ell}
$$

by (5.3). When $0<j<k-1$, the above equation can be rewritten recursively to give:

$$
s_{j k}=s_{(j-1) k}+s_{j(k-1)}+s_{(j-1)(k-1)} .
$$

Like before, we can create a triangular array of integers where $k$ indexes the rows $(k \geq 1)$ and $j$ indexes the columns $(0 \leq j<k)$. The diagonal entries of the triangle give the values of $s_{i}$. Like before, we define $s_{00}=1$ and $s_{j j}=s_{(j-1) j}$ for $j>0$. Now, this new triangular array is known as the super-Catalan triangle. One property of this triangle is that the sequence of diagonal entries is the sequence of super-Catalan numbers, also known as the little Schröder numbers [4]. Multiplying the little Schröder numbers by two and reindexing gives the Schröder numbers.

Combining (5.6) and (5.7), we have:

$$
\Omega(n)=\Omega(n, 0)+\Omega(n, 2)+\left(c_{n-2}+s_{n-2}\right)+\sum_{k=1}^{n-3}\left(c_{k}+s_{k}\right) \Omega(n-k, 2) .
$$

Next, by applying (5.1) and rearranging terms, we have: 


$$
\begin{aligned}
\Omega(n)= & \Omega(n-1)+\sum_{k=2}^{n-1}\left(c_{k-1}+s_{k-1}\right) \Omega(n-k)+\Omega(n, 0)-\Omega(n-2,0) \\
& -\sum_{k=3}^{n-1}\left(c_{k-2}+s_{k-2}\right) \Omega(n-k, 0) .
\end{aligned}
$$

Finally, by applying (4.1) and rearranging terms, we have (1.4).

Let $\mathcal{F}(z)=\sum_{n=0}^{\infty} \Omega(n) z^{n}$ be the generating function of $\Omega$, for $p=2$. Using the usual generating function calculations, including the generating functions for the Catalan and Schröder numbers, one computes that:

$$
\mathcal{F}(z)=\frac{\left(2-z-\sqrt{1-4 z}-\sqrt{1-6 z+z^{2}}\right)(1-z)+2\left(z^{2}-1\right)}{\left(2-z-\sqrt{1-4 z}-\sqrt{1-6 z+z^{2}}\right)\left(1-z-z^{2}\right)+2\left(z^{3}+z^{2}+z-1\right)} .
$$

Like in the case of odd primes, a non-recursive formula for this sequence is too complicated and impractical and hence is omitted.

Acknowledgements The contents of this paper were derived from the author's doctoral dissertation [16], written under the supervision of Stephen P. Humphries, that was submitted to Brigham Young University. All computations made in preparation of this paper were accomplished using the computer software Magma [1], and the exact code can be found in [7,16], the second reference being the master's thesis of Brent Kerby, another student of Humphries. Helpful suggestions, instructions, and guidance were offered to the author by Michael Barrus on many of combinatorical topics in this paper. Acknowledgment should also be given by Petr Vojtechovsky for his insights into how the topics of this paper related to the PORC conjecture. Finally, the author would like to thank the referees for their helpful comments and suggestions.

\section{References}

1. Bosma, W., Cannon, J., Playoust, C.: The Magma algebra system. I. The user language. J. Symbolic Comput. 24(3-4), 235-265 (1997). Computational algebra and number theory (London, 1993)

2. du Sautoy, M., Vaughan-Lee, M.R.: Non-PORC behaviour of a class of descendant $p$-groups. J. Algebra 361, 287-312 (2012)

3. Evseev, A.: Higman's PORC conjecture for a family of groups. Bull. Lond. Math. Soc. 40(3), 415-431 (2008)

4. Fischer, J.: Super-Catalan triangle. The On-Line Encyclopedia of Integer Sequences (2008). http:// oeis.org/A144944

5. Higman, G.: Enumerating p-groups. I: inequalities. Proc. Lond. Math. Soc. 10(3), 24-30 (1960)

6. Higman, G.: Enumerating p-groups. II: problems whose solution is PORC. Proc. Lond. Math. Soc. 10(3), 566-582 (1960)

7. Kerby, B.: Rational Schur rings over Abelian groups. Master's thesis, Brigham Young University (2008)

8. Klin, M.K., Pöschel, R.: The Konig problem, the isomorphism problem for cyclic graphs and the method of Schur rings. Algebr. Methods Graph Theory 1, 2 (1978)

9. Kovács, I.: The number of indecomposable Schur rings over a cyclic 2-group. Sém. Lothar. Combin. 51, 5 (2005)

10. Leung, K.H., Ma, S.L.: The structure of Schur rings over cyclic groups. J. Pure Appl. Algebra 66, 287-302 (1990)

11. Leung, K.H., Man, S.H.: On Schur rings over cyclic groups II. J. Algebra 183, 273-285 (1996)

12. Leung, K.H., Man, S.H.: On Schur rings over cyclic groups. Israel J. Math. 106, 251-267 (1998) 
13. Liskovets, V., Pöschel, R.: Counting circulant graphs of prime-power order by decomposing into orbit enumeration problems. Discrete Math. 214, 173-191 (2000)

14. Ma, S.L.: On association schemes, Schur rings, strongly regular graphs and partial difference sets. Ars Combin. 21, 211-220 (1989)

15. Meeussen, W.: Catalan's triangle. The On-Line Encyclopedia of Integer Sequences (2008). http://oeis. org/A009766

16. Misseldine, A.: Algebraic and combinatorial properties of Schur rings over cyclic groups. Ph.D. thesis, Brigham Young University (2014)

17. Muzychuk, M.: The structure of Schur rings over cyclic groups of square-free order. Acta Appl. Math. 52, 163-181 (1998)

18. Muzychuk, M.E.: The structure of rational Schur rings over cyclic groups. European J. Combin. 14, 479-490 (1993)

19. Muzychuk, M.E.: On the structure of basic sets of Schur rings over cyclic groups. J. Algebra 169, 655-678 (1994)

20. Newman, M.F., O’Brien, E.A., Vaughan-Lee, M.R.: Groups and nilpotent Lie rings whose order is the sixth power of a prime. J. Algebra 278(1), 383-401 (2004)

21. O'Brien, E.A., Vaughan-Lee, M.R.: The groups with order $p^{7}$ for odd prime $p$. J. Algebra 292(1), 243-258 (2005)

22. Wielandt, H.: Zur theorie der einfach transitiven permutationsgruppen II (German). Math. Z. 52, 384393 (1949)

23. Witty, B.E.: Enumeration of groups of prime-power order. Bull. Aust. Math. Soc. 76, 479-480 (2007)

24. Ziv-Av, M.: Enumeration of Schur rings over small groups. In: Proceedings of 16th International Workshop on Computer Algebra in Scientific Computing, CASC 2014, Warsaw, Poland, September 8-12, 2014, pp. 491-500. Springer, Berlin (2014)

Publisher's Note Springer Nature remains neutral with regard to jurisdictional claims in published maps and institutional affiliations. 